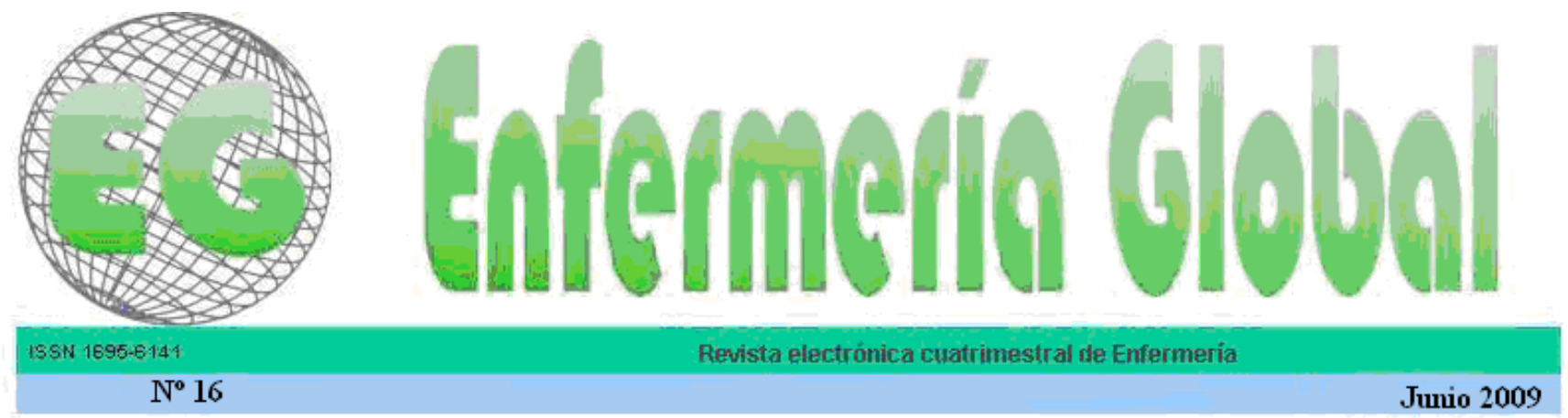

www.um.es/egloball

\title{
CLÍNIGA
}

\section{EL AUMENTO DE LA INDEPENDENCIA DE LOS PACIENTES EN EL HOSPITAL}

THE INCREASE OF PATIENT INDEPENDENCE IN HOSPITAL

*Blanca Gutiérrez, JJ., "Caraballo Núñez, MA., ${ }^{* *}$ Luque Pérez, M., ${ }^{* * *}$ Muñoz Segura, R.

*Supervisor. Hospital de Montilla.**Auxiliar de Enfermería. Hospital de Alta Resolución de Puente Genil. ***Enfermero. Hospital de Montilla. Córdoba

Palabras clave: Enfermera, independencia, actividades básicas de la vida diaria, investigación de resultados en salud, hospital

Keywords: Nurse, independence, Basic Activities of daily living, health outcomes research, hospital.

\section{RESUMEN}

Introducción: Aumentar el nivel de independencia de nuestros pacientes, es una premisa constante en la ejecución de nuestros cuidados y además ha servido de fundamento para algunas de las teorías que han dado forma a nuestro marco conceptual. El objetivo de nuestro trabajo es determinar como varía el nivel de independencia de los pacientes tras un período de ingreso hospitalario.

Metodología: Estudio de tipo descriptivo y analítico. La población de referencia son los pacientes ingresados en el Hospital de Montilla (Córdoba) incluídos en los procesos asistenciales de tipo Médico o Traumatológico.

Resultados: realizamos contraste de hipótesis para comprobar si hay diferencias estadísticamente significativas entre el índice de Barthel al ingreso y el índice de Barthel al alta. Empleamos la prueba paramétrica de la t de Student para grupos apareados. El nivel de independencia aumenta tanto en los pacientes incluídos en los procesos asistenciales de tipo Médico (5,93 IC 95\%: 3,64-8,22; $\mathrm{p}<0,0005)$ como Traumatológico (18,69 IC 95\%: 14,06-23,32; $\mathrm{p}<0,0005)$. En los procesos de tipo Médico, a mayor estancia hospitalaria del paciente, el aumento de la independencia es mayor (aunque la correlación es débil).

Discusión: reseñamos los resultados de diversos estudios que han estudiado la dependenciaindependencia de los pacientes en diversos contextos: hospitales, unidades de enfermería, centros geriátricos. 


\section{ABSTRACT}

Introduction: Increasing the level of our patients' independence is a constant premise in the execution of our care and also has served as the basis for some of the theories that have given form to our conceptual framework. The target of our work is to determine how the patients' level of independence changes after a period of hospital stay.

Methodology: Descriptive and analytic study. The reference population is the patients entered in the Hospital of Montilla, Córdoba included in the assistance processes of the Medical or Orthopedic Departments.

Results: We made a hypothesis contrast to check if there were statistically significant differences among the Barthel scale at hospital check in and the Barthel scale at hospital check out. We used the parametric test of the t of Student for paired groups. The level of independence increases as much in the patients included in the assistance processes of Medical Department (5.93 IC 95\%: 3.64-8.22; $\mathrm{p}<0.0005)$ as Orthopedic Department (18.69 IC $95 \%$ : 14.06-23.32; $p<0.0005)$. In Medical processes, the increase of the independence is greater when the patients' stay is longer (although the correlation is weak).

Discussion: We review the results of diverse studies that have studied the dependency-independence of patients in diverse contexts: Hospitals, Nursing Units, and Geriatric Centers.

\section{INTRODUCCIÓN}

Aumentar el nivel de independencia de los pacientes a nuestro cargo es una premisa constante en la ejecución de nuestros cuidados y además ha servido de fundamento para algunas de las teorías que han dado forma al marco conceptual de nuestra profesión. De hecho, conseguir el menor grado de dependencia posible de las poblaciones es un objetivo que compartimos las enfermeras con otras disciplinas científicas, ya que la dependencia, la podemos considerar como un sinónimo de la discapacidad.

La dependencia de tipo físico o funcional, es definida por Aguilar Hernández et al como "la dificultad para realizar una o más de las Actividades Básicas de la Vida Diaria (ABVD): comer, lavarse, vestirse y desvestirse, arreglarse, deposiciones, micción, uso del retrete, trasladarse, deambular y subir y bajar escalones" (1). Estas 10 actividades junto con el conocido índice de Barthel basado en las mismas, fueron reflejadas por Mahoney y Barthel en una publicación que data de 1965 (2), si bien este índice ya se usaba desde unos años antes en algunos hospitales de pacientes crónicos del estado de Maryland en Estados Unidos. (3)

Por lo tanto, el índice de Barthel, trata de aproximarnos cuantitativamente al nivel de dependencia-independencia de un paciente, según como sea su realización de las ABVD. Cada una de estas 10 actividades se valora de forma independiente otorgándole una puntuación de $0,5,10$ ó 15 puntos, obteniéndose una puntuación total que puede variar desde 0 (dependencia total) a 100 (independencia). (3)

Cid Ruzafa y Damián Moreno realizaron una exhaustiva revisión bibliográfica en la que nos muestran, entre otros muchos aspectos, cuáles son las características de fiabilidad, validez y sensibilidad de este instrumento, concluyendo lo siguiente: "El Indice de Barthel es una medida de la discapacidad física con demostrada validez y fiabilidad, fácil de aplicar y de interpretar y cuyo uso rutinario es recomendable". (3) 
La recuperación o el mantenimiento de la independencia funcional es un resultado de salud que puede ser abordado desde varias perspectivas:

- Por un lado, forma parte de los resultados en salud catalogados como clásicos (calidad de vida, mortalidad, morbilidad, satisfacción, resultados de las pruebas clínicas...). Concretamente, formaría parte de los resultados determinantes de la calidad de vida, que serían la funcionalidad física, la funcionalidad psíquica y la social (4).

- Pero, por supuesto, también se trata de unos de los denominados resultados sensibles a la práctica enfermera, que se definen como "estado, conducta o percepción de un individuo, familia o comunidad medida a lo largo de un continuo en respuesta a intervención(es) de enfermería" (5). Por lo tanto, la dependencia-independencia del paciente también entra a formar parte de los lenguajes estructurados de medición de resultados enfermeros como la Clasificación Internacional para la Práctica Enfermera (Internacional Classification for Nursing Practice) o la Clasificación de Resultados de Enfermería de lowa (Nursing Outcomes Classification). (6)

El objetivo de nuestro trabajo es determinar cómo varía el nivel de independencia de los pacientes tras un período de ingreso hospitalario. Para ello estudiaremos la evolución del citado índice de Barthel entre el momento del ingreso y del alta del Hospital.

\section{METODOLOGÍA}

Realizamos un estudio de tipo descriptivo y analítico.

Mediante historias clínicas recuperadas al azar, hemos recogido como datos el índice de Barthel de los pacientes en el momento del ingreso y en el momento del alta (antes y después de aplicar los planes de cuidados). El período de estudio considerado ha sido desde el mes de abril del año 2007 hasta el mes de marzo de 2008. Los planes de cuidados utilizados están basados en los planes de cuidados estandarizados de los procesos asistenciales integrados del Sistema Sanitario Público de Andalucía. (7)

La población de referencia son los pacientes ingresados en el Hospital de Montilla (Córdoba) incluidos en los procesos asistenciales de tipo Médico o Traumatológico.

Para realizar el análisis de datos hemos utilizado el programa SPSS v 11.5.

\section{RESULTADOS}

\section{Procesos asistenciales de tipo médico:}

Cálculo del tamaño de la muestra: si aceptamos un riesgo a de 0.05 y un riesgo $\beta$ de 0.20 en un contraste bilateral, necesitamos los datos de 86 pacientes para detectar una diferencia igual o superior a 10 unidades. Hemos asumido una desviación estándar de 33. Hemos estimado una proporción de pérdidas de seguimiento del $0 \%$.

En la tabla I recogemos las características de los pacientes incluidos en la muestra. 
Tabla I. Características de la muestra. Procesos de tipo médico

\begin{tabular}{|l|r|}
\hline Sexo & \\
\hline hombre & 51,2 \\
\hline mujer & 48,8 \\
\hline Cuidador principal & 29,1 \\
\hline pareja & 50 \\
\hline hijos & 0 \\
\hline padres & 0 \\
\hline hermanos & 5,8 \\
\hline otros familiares & 0 \\
\hline veciones o amigos & 1,2 \\
\hline sin cuidador & 14 \\
\hline residencia & 79,76 (DE 9,117) \\
\hline Edad media & 6,43 (DE 5,604) \\
\hline Estancia media & 29,53 (DE 32,563) \\
\hline Barthel al ingreso medio & 35,47 (DE 33,743) \\
\hline Barthel al alta medio & \\
\hline
\end{tabular}

A continuación realizamos contraste de hipótesis para comprobar si hay diferencias estadísticamente significativas entre el índice de Barthel al ingreso y el índice de Barthel al alta. Empleamos la prueba paramétrica adecuada para comparar una variable cuantitativa medida en dos grupos apareados, la $t$ de Student. Obtenemos que en los pacientes de nuestro Hospital incluidos en los procesos de tipo médico se produce un aumento medio del índice de Barthel de 5,93 puntos (IC 95\%: 3,64-8,22; $p<0,0005$ ). En la figura 1, representamos estas diferencias mediante un diagrama de barras de error. 
Figura 1. Barras de error. Barthel al ingreso frente al Barthel al alta. Procesos médicos.

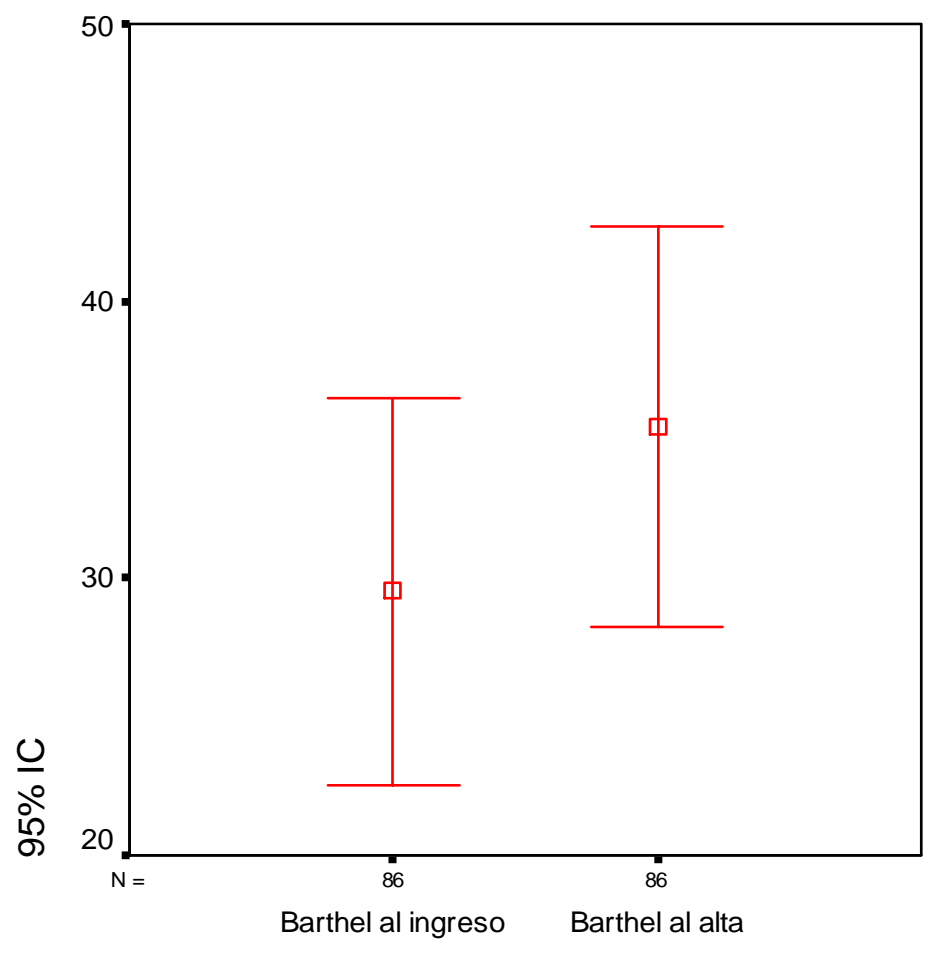

Si calculamos el coeficiente de correlación de Pearson entre el Barthel al ingreso y el Barthel al alta obtenemos un valor de 0,949 $(p<0,0005)$. Por lo tanto podemos representar una recta de regresión y calcular la ecuación de dicha recta $\left(y=6,4341+0,9829 x ; R^{2}=0,900\right)$. (Figura 2) 
Figura 2. Recta de regresión. Barthel al ingreso frente al Barthel al alta. Procesos médicos.

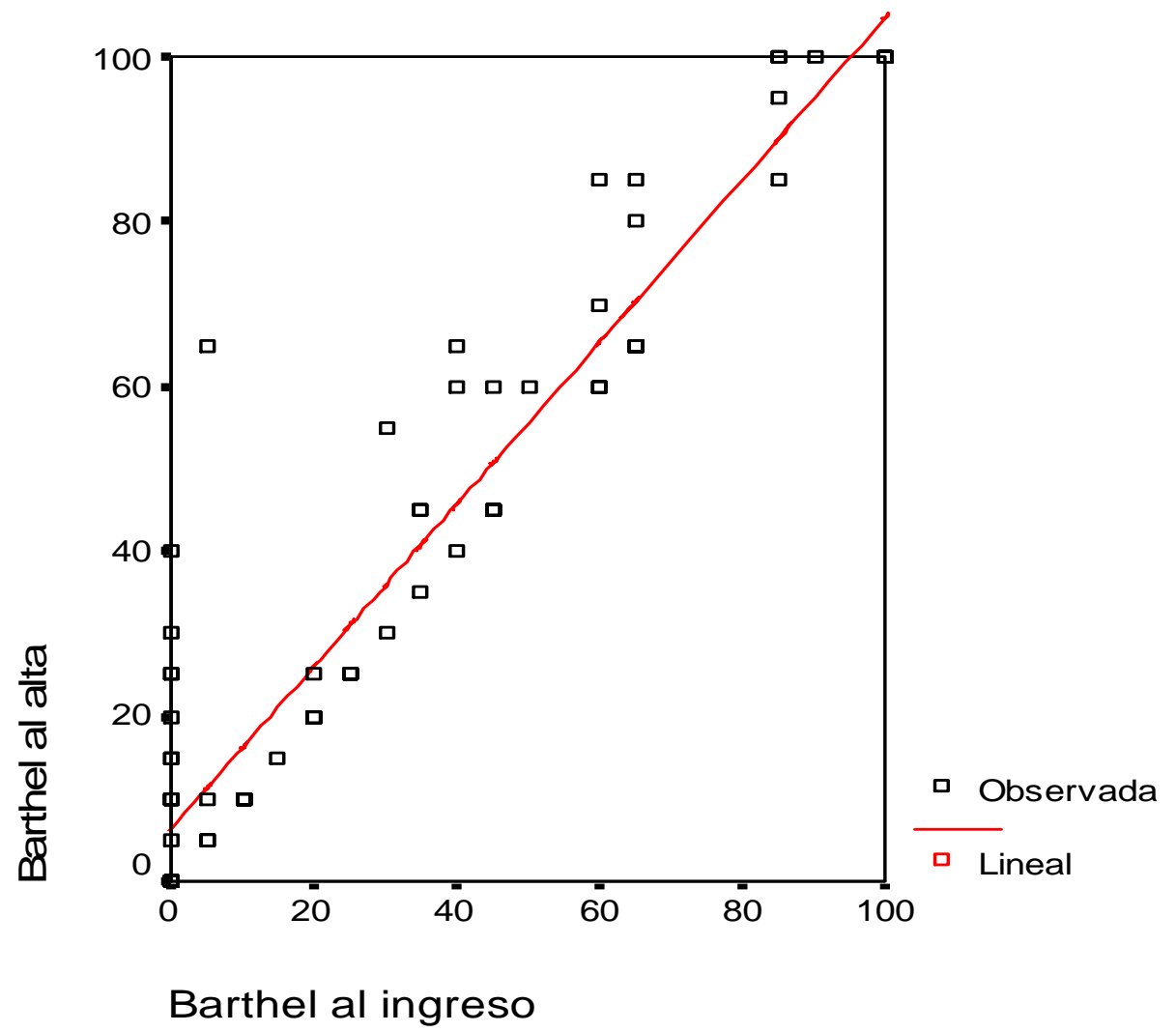

Hemos creado una nueva variable, la diferencia del Barthel al ingreso y al alta y hemos calculado el coeficiente de correlación de Pearson de esta variable para los días de estancia, la edad y el Barthel al ingreso. Sólo en el caso de los días de estancia, esta correlación es estadísticamente significativa $(r=0,268 ; p<0,05)$, pero al ser $r<0,6$ no podemos calcular una ecuación de regresión. Este cálculo nos indica que, en cierto grado, hay relación lineal entre la duración de la estancia hospitalaria y el aumento del nivel de independencia de los pacientes (a mayores estancias mayor aumento del Barthel), pero esto no significa que haya causalidad entre ambas variables.

En la tabla II resumimos los cálculos realizados para estas correlaciones. 
Tabla II. Cálculo de coeficientes de correlación. Procesos médicos

\begin{tabular}{|l|lr|r|r|r|r|}
\hline $\begin{array}{l}\text { variable } \\
\text { independiente }\end{array}$ & $\begin{array}{l}\text { variable } \\
\text { dependiente }\end{array}$ & \multicolumn{1}{l|}{$\boldsymbol{r}$} & p & recta de regresión & $\mathbf{R}^{2}$ & \\
\hline barthel al ingreso & barthel al alta & 0,949 & 0,000 & $\mathrm{y}=6,4341+0,9829 x$ & 0,900 \\
\hline días de estancia & $\begin{array}{l}\text { aumento del } \\
\text { barthel }\end{array}$ & 0,268 & 0,013 & & \\
\hline edad & $\begin{array}{l}\text { aumento del } \\
\text { barthel }\end{array}$ & 0,013 & $0,904 . \mathrm{NS}$ & & & \\
\hline barthel al ingreso & $\begin{array}{l}\text { aumento del } \\
\text { barthel }\end{array}$ & $-0,052$ & $0,635 . \mathrm{NS}$ & & & \\
\hline
\end{tabular}

\section{Procesos asistenciales de tipo traumatológico:}

Cálculo del tamaño de la muestra: si aceptamos un riesgo $\alpha$ de 0.05 y un riesgo $\beta$ de 0.20 en un contraste bilateral, necesitamos los datos de 42 pacientes para detectar una diferencia igual o superior a 10 unidades. Hemos asumido una desviación estándar de 23. Hemos estimado una proporción de pérdidas de seguimiento del $0 \%$.

En la tabla III recogemos las características de los pacientes incluidos en la muestra.

Tabla III. Características de la muestra. Procesos de tipo traumatológico

\begin{tabular}{|c|c|}
\hline \multicolumn{2}{|l|}{ Sexo } \\
\hline hombre & 42,9 \\
\hline mujer & 57,1 \\
\hline \multicolumn{2}{|l|}{ Cuidador principal } \\
\hline pareja & 40,5 \\
\hline hijos & 47,6 \\
\hline padres & 0 \\
\hline hermanos & 0 \\
\hline otros familiares & 4,8 \\
\hline veciones o amigos & 0 \\
\hline sin cuidador & 0 \\
\hline residencia & 7,1 \\
\hline Edad media & 78,02 (DE 8,438) \\
\hline Estancia media & 7,81 (DE 6,021) \\
\hline Barthel al ingreso medio & 51,19 (DE 23,291) \\
\hline Barthel al alta medio & 69,88 (DE 25,363) \\
\hline
\end{tabular}


Utilizamos el mismo proceso que hemos empleado para los pacientes incluidos en los procesos asistenciales de tipo médico. El resultado también es favorable y podemos afirmar que en los pacientes de nuestro Hospital incluidos en los procesos de tipo traumatológico se produce un aumento medio del índice de Barthel de 18,69 puntos (IC 95\%: 14,06-23,32; $\mathrm{p}<0,0005)$. En la figura 3, representamos estas diferencias mediante un diagrama de barras de error.

Figura 3. Barras de error. Barthel al ingreso frente al Barthel al alta. Procesos traumatológicos

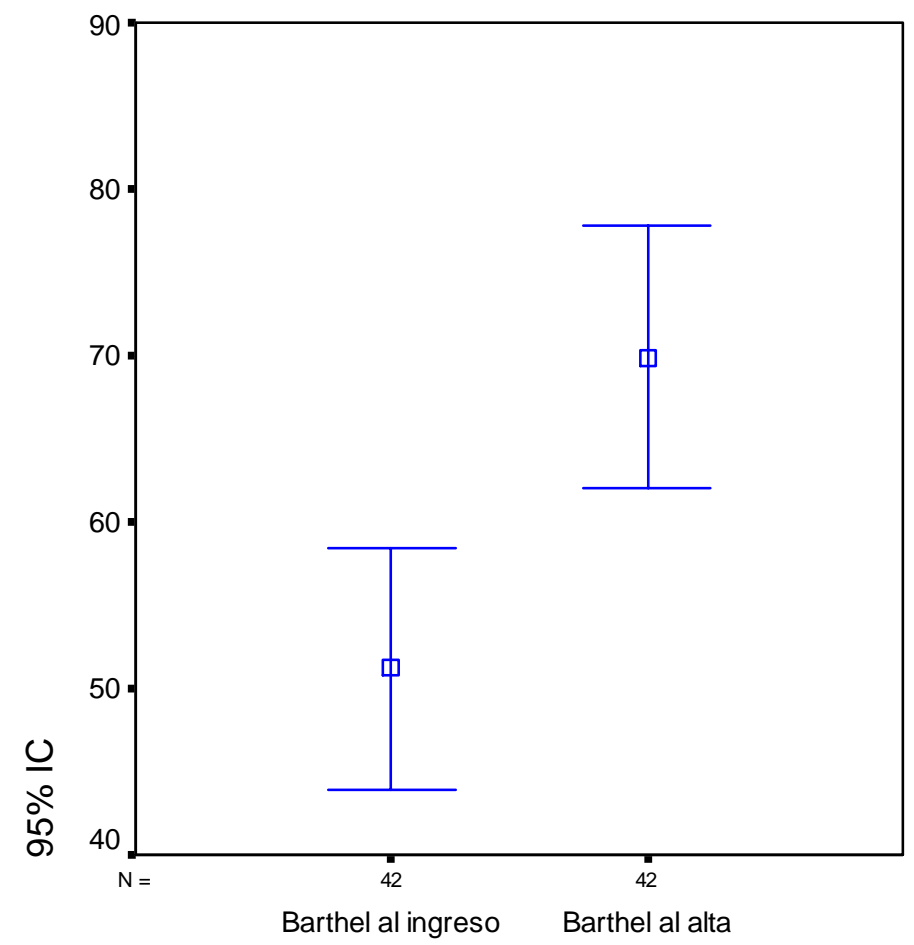

Como en el apartado anterior, calculamos el coeficiente de correlación de Pearson entre el Barthel al ingreso y el Barthel al alta y obtenemos un valor de $0,817(p<0,0005)$. Por lo tanto podemos representar una recta de regresión y calcular la ecuación de dicha recta $\left(y=24,3515+0,8894 x ; R^{2}=0,667\right)$. (Figura 4). 
Figura 4. Recta de regresión. Barthel al ingreso frente al Barthel al alta. Procesos traumatológicos

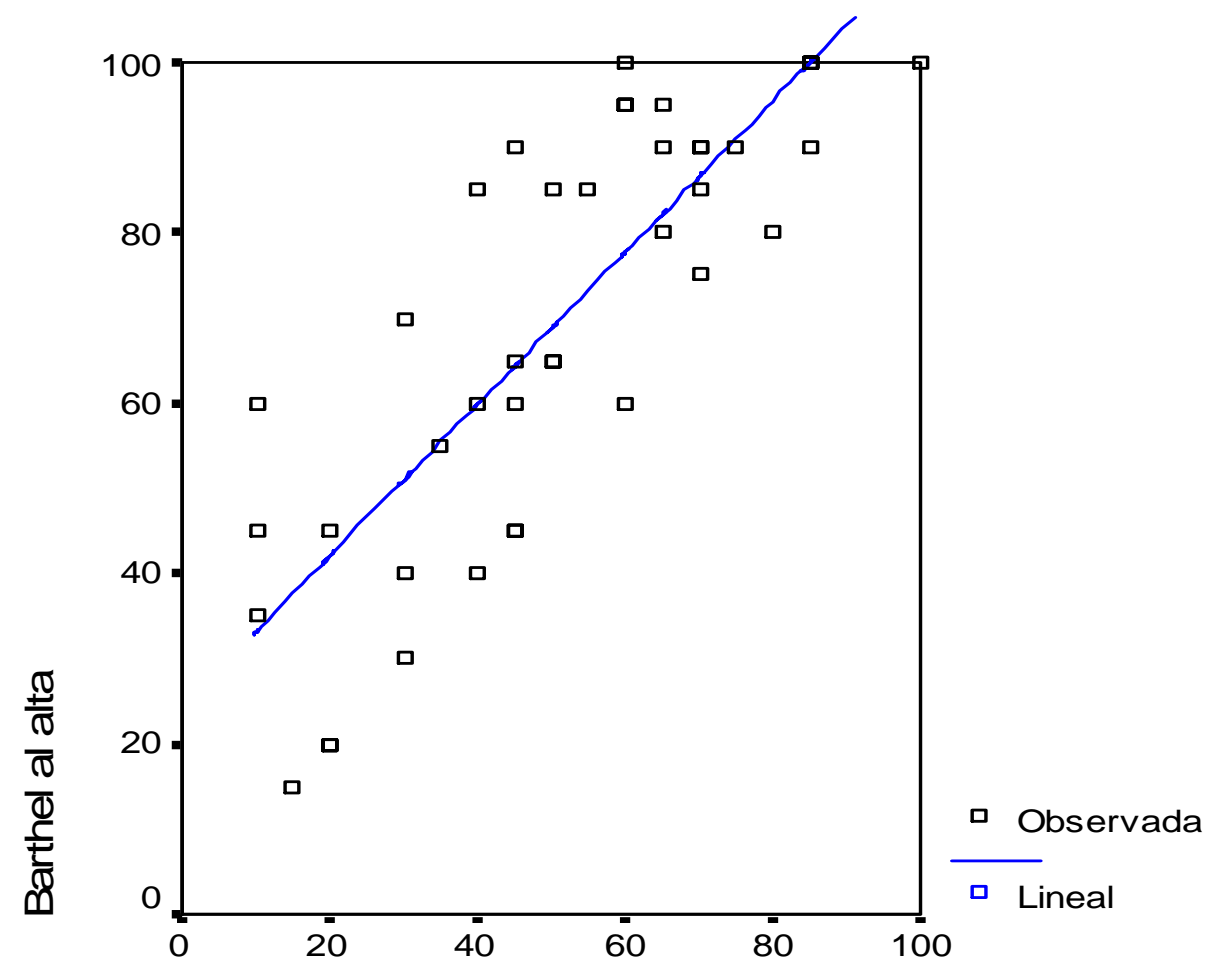

Barthel al ingreso

A continuación creamos la variable nueva calculada a partir de la diferencia del Barthel al ingreso y al alta para tratar de encontrar correlación con los días de estancia, la edad del paciente y el Barthel al ingreso de los pacientes. En ninguno de estos casos se obtiene una correlación estadísticamente significativa. Mostramos los cálculos en la tabla IV.

Tabla IV. Cálculo de coeficientes de correlación. Procesos traumatológicos

\begin{tabular}{|c|c|c|c|c|c|}
\hline $\begin{array}{l}\text { variable } \\
\text { independiente }\end{array}$ & $\begin{array}{l}\text { variable } \\
\text { dependiente }\end{array}$ & $r$ & $p$ & recta de regresión & $\mathbf{R}^{2}$ \\
\hline barthel al ingreso & barthel al alta & 0,817 & 0,000 & $y=24,3515+0,8894 x$ & 0,667 \\
\hline días de estancia & $\begin{array}{l}\text { aumento del } \\
\text { barthel }\end{array}$ & 0,272 & $0,081 . N S$ & & \\
\hline edad & $\begin{array}{l}\text { aumento del } \\
\text { barthel }\end{array}$ & $-0,260$ & $0,096 . N S$ & & \\
\hline barthel al ingreso & $\begin{array}{l}\text { aumento del } \\
\text { barthel }\end{array}$ & $-0,173$ & $0,272 . N S$ & & \\
\hline
\end{tabular}

\section{DISCUSIÓN}

Discutiremos a continuación los resultados de algunos trabajos que también han estudiado el grado de autonomía de los pacientes y que han utilizado para ello el índice de Barthel, en instituciones de tipo geriátrico, en unidades de tipo Unidad de Cuidados de Enfermería (UCE) o en el Hospital. 
Lobo y Pereira realizaron un estudio descriptivo con 164 ancianos institucionalizados de las ciudades de Braga y Oporto, en los que estudiaron su funcionalidad mediante diversos parámetros (medidas antropométricas, índice de Barthel, mini mental-state examination, functional fitness test). En sus resultados encontraron correlación estadísticamente significativa entre la dependencia y las variables edad, índice de masa corporal y número de enfermedades crónicas (8).

En nuestro trabajo, no hemos encontrado correlación entre la edad y la variación de la dependencia, es decir, que a mayor edad el aumento del Barthel fuera menor. Como hemos visto, el aumento de la independencia ha sido menor en los pacientes de los procesos de tipo Médico, que se trataban en la mayoría de los casos de enfermos pluripatológicos (con diversas enfermedades crónicas).

Rojas Ocaña et al estudiaron la autonomía de 19 ancianos de una residencia de Huelva mediante el índice de Barthel (actividades básicas de la vida diaria) y el índice de Lawton (actividades instrumentales de la vida diaria), medidos ambos a los 20 días del ingreso y a los 18 meses del mismo. La media del índice de Barthel evolucionó desde 92,36 puntos en el primer momento hasta los 84,21 puntos obtenidos en la segunda medición, alcanzándose una disminución estadísticamente significativa $(p=0,002)$. Las actividades instrumentales también bajaron desde una puntuación de 3,57 hasta 2,31 $(p<0,001)(9)$.

En nuestro estudio, el índice de Barthel por el contrario experimenta una subida estadísticamente significativa, aunque hemos de considerar la diferencia de contextos: una residencia geriátrica en el caso de Rojas Ocaña y un Hospital con una estancia media de unos 7 días en nuestro caso.

Aguilar Hernández et al desarrollaron un estudio de tipo observacional con 45 pacientes "adultos mayores" en México. Encontraron valores mayores de dependencia funcional para las mujeres (1). Nosotros no hemos estudiado la dependencia-independencia en función del sexo.

Jiménez Navascués e Híjar Ordovas estudiaron la influencia de las alteraciones visuales en la independencia de una población de 79 ancianos de una residencia de Soria. En sus resultados encontraron que las alteraciones visuales influyen en la autonomía (con valores estadísticamente significativos) (10). Encontraron relación entre la edad y el mayor nivel de dependencia (como ocurría en el estudio de Lobo y Pereira). No hallaron relación entre el período de estancia del anciano y el aumento de la dependencia, mientras que en los pacientes de nuestra investigación incluidos en los procesos de tipo médico sí se produce esta relación aunque a la inversa (a mayor estancia mayor aumento de la independencia). De nuevo hemos de tener en cuenta de que se trata de contextos muy diferentes (residencia-hospital).

Pérez Fonollá y García Castillo estudiaron de manera descriptiva la capacidad funcional y la percepción de independencia en una muestra de 20 ancianos hospitalizados en el Hospital de San Rafael (Granada). No encontraron apenas diferencias entre la percepción subjetiva de funcionalidad y la que se midió de manera objetiva (11). En nuestra investigación no hemos incluido el estudio de la funcionalidad desde el punto de vista subjetivo del paciente.

Álvarez Plaza et al estudiaron la evolución del índice de Barthel en una muestra de 135 pacientes críticos sometidos a ventilación mecánica del Hospital de Alcorcón (Madrid), midiendo la escala en tres tiempos: al ingreso en la $\mathrm{UCl}$, al mes y transcurrido un año del 
alta. Los pacientes que ingresaron con menores niveles de independencia eran también los menos independientes al mes del ingreso en la unidad. Transcurrido un año del ingreso la mayoría de los pacientes habían recuperado sus niveles funcionales previos a la estancia en la $\mathrm{UCI}$ (12). Al mes del ingreso en la Unidad los pacientes habían experimentado una disminución significativa del Barthel, mientras que en nuestro estudio la estancia hospitalaria ha aumentado la independencia, pero los resultados son poco comparables debido a que los pacientes en uno y otro caso son muy distintos (pacientes sometidos a ventilación mecánica en una UCl-pacientes traumatológicos y médicos ingresados en una planta de Hospitalización).

Alonso Pérez et al realizaron un estudio descriptivo en el que estudiaron el nivel de independencia de 161 pacientes mayores de 65 años ingresados en el Hospital de Mendaro (Guipúzcoa). Hallaron diferencias significativas entre las unidades de Medicina Interna y Cirugía para los ítem de comer y deposición (13). En nuestros resultados el aumento de la independencia es mayor en los pacientes de los procesos asistenciales de tipo traumatológico con respecto a los de tipo médico.

Mesa Fernández et al desarrollaron un estudio descriptivo con una muestra de 32 pacientes de la Unidad de Cuidados de Enfermería del Hospital Dr. Sagaz de Jaén, en el que analizaron el Barthel al ingreso y al alta de estos pacientes. Obtuvieron una evolución del índice de Barthel desde una media de 45,94 puntos al ingreso hasta una media de 78,28 puntos al alta ( $p=0$ '0001), con una estancia media de 89 días (14). Desde esta misma unidad, Díaz Castro et al nos presentaron resultados igual de favorables en un estudio realizado entre los años 2003 y 2004 (15). Nuestro estudio también evidencia el aumento de la independencia de los pacientes, esta vez también en un entorno hospitalario pero con estancias de mucha menor duración.

\section{CONCLUSIÓN}

Como evidencian los resultados de nuestro trabajo, el ingreso en un Hospital no tiene porque producir una reducción de la funcionalidad de los pacientes, al contrario, su estancia puede y debe significar un aumento de sus capacidades y de su nivel de independencia.

Nuestro estudio apunta incluso una leve relación positiva entre la duración de la estancia y el aumento de la independencia en el caso de los pacientes incluidos en procesos de tipo médico. Pero estas conclusiones deben ser completadas con las aportaciones de futuras investigaciones ubicadas en otros Hospitales y unidades de distintos ámbitos.

\section{BIBLIOGRAFÍA}

1. Aguilar Hernández, RM; Félix Alemán, A; Martínez Aguilar, ML; Vega Alanís, MC; Quevedo Díaz, MM; Gutiérrez Sánchez, G. Dependencia funcional y género del adulto mayor. Desarrollo Científ Enferm -Méx- 2007 nov-dic 15(10):436-439.

2. Mahoney FI, Barthel DW. Functional evaluation: the Barthel Index. Md Med J 1965; 14: 61-65.

3. Cid-Ruzafa J, Damián-Moreno J. Valoración de la discapacidad física: El índice de Barthel. Rev. Española de Salud Pública, 1997:127-137. 
4. Morales Asencio JM. Investigación de resultados en enfermería. Index Enferm 2004 primavera-verano, año XIII (44-45):37-41.

5. Johnson M, Maas M, Moorhead S. Clasificación de Resultados de enfermería, 3aㅗ Edición. Madrid Elsevier España 2005.

6. Sebastián Viana, T; Gil Hernández, MR. Resultados sensibles a la práctica enfermera. Una reflexión sobre la situación actual. Rev Tesela 2008; 3.

7. Planes de cuidados estandarizados de los procesos asistenciales integrados. Página Web de la Consejería de Salud de la Junta de Andalucía. Disponible en http://www.csalud.junta-andalucia.es/procesos/listados.asp?sellista=4\&f=p.

8. Lobo, A; Pereira, Adriana. Idoso Institucionalizado: Funcionalidade e Aptidão Física. Referência 2007 jun II (4):61-68.

9. Rojas Ocaña, MJ; Torronjo Gómez, A; Rodríguez Ponce, C; Rodríguez Rodríguez, JB. Autonomía y estado de salud percibidos en ancianos institucionalizados. Gerokomos 2006 ene-mar 17(1):6-23.

10. Jiménez Navascués, L; Hijar Ordovas, CA. Los ancianos y las alteraciones visuales como factor de riesgo para su independencia. Gerokomos 2007 ene-mar 18(1):16-23.

11. Pérez Fonollá, MD; García Castillo, N. Percepción de independencia funcional en el anciano hospitalizado Nursing -Ed esp- 2006 feb 24(2):56-60.

12. Álvarez Plaza, G; Pérez Serna, Y; García Arias, M; Peñalta Sánchez, RM; Gordo Vidal, F. Variación de la calidad de vida al año de alta del ingreso en UCl y ventilación mecánica. Enferm Intensiva 2004 ene-mar 15(1):17-23.

13. Alonso Pérez, L; Ezkurra Loyoa, A; Amilibia Garaizabal, L; González Ignacio, A; Guridi Amenabar, M. Nivel de dependencia de los pacientes ingresados. Metas Enferm 2006 oct 9(8):18-24.

14. Mesa Fernández, E; Torres Medina, S; Alejo Esteban, JA. Efectividad de las intervenciones enfermeras sobre pacientes y familiares en una unidad de cuidados de enfermería. Nure Inv 2007 mar-abr 4(27).

15. Díaz Castro, M; Garrido Palomino, MM; Camacho Carballo, B; Fernández Izquierdo, G; Lara Molina, F; Chica Palomo, MD. Unidad de Cuidados de Enfermería: UCE. Inquietudes 2005 may-ago XI (32):18-23. 